\title{
ULTRAFILTER INVARIANTS IN TOPOLOGICAL SPACES
}

\author{
BY \\ VICTOR SAKS ${ }^{1}$
}

\begin{abstract}
Let $m \geqslant \kappa_{0}$ and $X=\prod_{i \in I} X_{i}$. Then $X$ is $\left[\kappa_{0}, \mathfrak{m}\right]$-compact if and only if $\Pi_{i \in J} X_{i}$ is $\left[\kappa_{0}, m\right]$-compact for all $J \subset I$ with $|J|<2^{2^{m}}$. Let $m \geqslant \aleph_{0}$, $\left(x_{\xi}: \xi<\mathfrak{m}\right)$ a net in $X, p \in X$, and $\mathscr{D} \in \beta(\mathfrak{m})$. Then $p=\mathscr{D}-\lim _{\xi<\mathfrak{m}} x_{\xi}$ if $\left\{\xi<\mathfrak{m}: x_{\xi} \in U\right\} \in \mathscr{D}$ for every neighborhood $U$ of $p$. Every topological space is characterized by its $\mathscr{D}$-limits. Several topological properties are described using ultrafilter invariants, including compactness and perfect maps. If $X$ is a Hausdorff space and $D$ is a discrete space equipotent with a dense subset of $X$, then $X$ is a continuous perfect image of a subspace of $\beta D$ which contains $D$ if and only if $X$ is regular.
\end{abstract}

1. Introduction. In $\left[\mathbf{B}_{\mathbf{1}}\right], \mathbf{A}$. Bernstein makes the following definitions.

Let $\mathcal{D}$ be an ultrafilter over the positive integers $N$, let $X$ be a topological space, $\left(x_{n}: n \in N\right)$ a sequence of points of $X$, and $x \in X$. Then $x$ is a $\mathscr{D}$-limit point of $\left(x_{n}: n \in N\right)$ provided that if $U$ is open in $X$ with $x \in U$, then $\{n$ : $\left.x_{n} \in U\right\} \in \mathscr{D}$; in this case we write $x=\mathscr{D}-\lim _{n \rightarrow \infty} x_{n}$. If every sequence in $X$ has a $\mathscr{D}$-limit point, then $X$ is $\mathscr{D}$-compact.

In [GS], John Ginsburg and the present author showed that a Hausdorff space $X$ has all of its powers countably compact if, and only if, it is Q -compact, for some $\mathscr{D}$ in $\beta N \backslash N$.

In this paper, we generalize the notion of $D$-limit to nets indexed by an arbitrary infinite cardinal. We show that any topological space is characterized by its generalized $\mathscr{D}$ - limits, and that a number of topological properties can be described by the use of ultrafilter invariants.

It should be noted that the concept of $\mathscr{D}$-limit was used in other guises by several authors prior to the appearance of Bernstein's paper. The most noteworthy example is $\mathbf{Z}$. Frolik's nice Sums of ultrafilters $\left[\mathbf{F}_{\mathbf{1}}\right]$ paper. When Frolik writes $x=\Sigma y\left\{x_{n}\right\}$, this means precisely that $x=y-\lim x_{n}$. Also, in the present author's thesis $\left[\mathbf{S}_{\mathbf{1}}\right]$, the notion of $\mathscr{D}$-limit appears in the rather

Presented to the Society, January 26, 1975; received by the editors February 2, 1976.

AMS (MOS) subject classifications (1970). Primary 54A20, 54B10, 54C10, 54D10, 54D20, 54D30; Secondary 54A25.

Key words and phrases. Ultrafilter, $\mathscr{D}$-limit point, $\mathscr{D}$-compact, countably compact product, $[\mathfrak{n}, \mathfrak{m}]$-compact, complete accumulation point, $C[\mathfrak{n}, \mathfrak{m}]$, perfect map, regular space.

1 The author would like to thank the University of Costa Rica for support of his trip to the International Congress of Mathematicians in Vancouver, which served to stimulate much of this research. He is also grateful to Professor W. W. Comfort for his advice and encouragement concerning the writing of this paper. 
cumbersome form in completely regular Hausdorff spaces as follows: $\left(x_{n}\right.$ : $n \in N)$ is a sequence in $X$ and if $i: N \rightarrow\left\{x_{n}: n \in N\right\}$ is the natural map, then $i^{\beta}(\mathscr{D})=x$.

Recently, assuming the continuum hypothesis, M. Rajagopalan and R. G. Woods [RW] constructed a sequentially compact space $X$ which is not $\mathscr{D}$-compact for any $\mathscr{D}$ in $\beta N \backslash N$. Thus $X^{2 c}$ is not countably compact, answering in the negative a question in $\left[\mathbf{S S}_{2}\right]$.

This question was then answered in the negative by several mathematicians using a variety of set-theoretic assumptions. In [ $\left.\mathbf{K}_{\mathbf{1}}\right]$, Kenneth Kunen, using at least the continuum hypothesis, constructed a first countable sequentially compact space which is not $\mathscr{D}$-compact for any $\mathscr{D}$. Jerry Vaughan $\left[\mathbf{V}_{\mathbf{3}}\right]$, using the set-theoretic hypothesis $\diamond$, has constructed a family of perfectly normal sequentially compact spaces whose product is not countably compact. See $\left[\mathbf{V}_{\mathbf{3}}\right]$ for an up-to-date discussion of attempts to answer the question in $\left[\mathbf{S S}_{\mathbf{2}}\right]$ without any additional set-theoretic assumptions.

For some interesting remarks concerning ultrafilters on $N$ as limit operators in compact Hausdorff spaces and for some very powerful results, see another paper by Kunen [ $\left.\mathbf{K}_{\mathbf{2}}\right]$.

We are grateful to Professors E. Michael, R. M. Stephenson, Jr., and Jerry E. Vaughan for some helpful comments and for supplying some references. Thanks are also due to the referee, whose careful reading of this paper saved us from several errors.

Definitions AND Notation. Throughout this paper the letters $\mathfrak{m}$ and $\mathfrak{n}$ will stand for infinite cardinals with $\mathfrak{n} \leqslant \mathfrak{m}$. As usual, the symbol $\mathfrak{n}^{+}$denotes the smallest cardinal greater than $n$.

Let $\left(x_{\xi}: \xi<\mathfrak{m}\right)$ be a transfinite sequence in $X, x \in X$, and $\mathscr{D} \in \beta(\mathfrak{m})$. Then $x$ is a $\mathscr{D}$-limit point of $\left(x_{\xi}: \xi<\mathfrak{m}\right)$ if $x \in U$ open implies that $\{\xi$ : $\left.x_{\xi} \in U\right\} \in \mathscr{D}$; in this case we write $x=\mathscr{D}-\lim _{\xi<m} x_{\xi}$.

$M^{\prime}=\{x \in X: x$ is an accumulation point of $M\}$.

A family $\left(a_{i}: i \in I\right)$ is faithfully indexed if $i_{1} \neq i_{2}$ implies that $a_{i_{1}} \neq a_{i_{2}}$. $\mu \mathrm{m}=\{\mathscr{D} \in \beta \mathfrak{m}: M \in \mathscr{D}$ implies $|M|=\mathfrak{m}\}$.

If $X$ is a space and $Y \subset X$, then a point $p$ of $X$ is a complete accumulation point of $Y$ provided that $|U \cap Y|=|Y|$ for each neighborhood $U$ of $p$ in $X$.

Note that we only use $z=\mathscr{D}-\lim _{\xi<m} x_{\xi}$ when $\mathscr{D} \in \beta(\mathfrak{m})$. If $\mathfrak{n} \leqslant \mathfrak{m}$, then since $\mu \mathrm{n} \subset \beta(\mathfrak{m})$ naturally, we can have $z=\mathscr{D}-\lim _{\xi<\mathfrak{m}} x_{\xi}$ and $\mathscr{D} \in$ $\mu$ n.

Easily checked facts, most of which are direct generalizations of results in [GS] are:

(i) if $\left(x_{\xi}: \xi<\mathfrak{m}\right)$ is faithfully indexed and $\mathscr{D} \in \mu \mathfrak{m}$, then if $x=\mathscr{D}-$ $\lim _{\xi<\mathfrak{m}} x_{\xi}$, then $x$ is a complete accumulation point of the set $\left\{x_{\xi}: \xi<\mathfrak{m}\right\}$; and conversely, if $x$ is a complete accumulation point of $\left\{x_{\xi}: \xi<\mathfrak{m}\right\}$, then 
there exists $\mathscr{D} \in \mu \mathrm{m}$ with $x=\mathscr{D}-\lim _{\xi<m} x_{\xi}$.

(ii) If $X$ is a Hausdorff space, then whenever the 9 -limit of a net exists, it is unique.

(iii) Let $X$ be a $T_{1}$ space. Let $\left(x_{\xi}: \xi<m\right)$ be a transfinite sequence and $x \in\left\{x_{\xi}: \xi<\mathfrak{m}\right\}^{\prime}$. Then there exists $\mathscr{D} \in \beta(\mathfrak{m}) \backslash \mathfrak{m}$ such that $x=\mathscr{D}-$ $\lim _{\xi<m} x_{\xi}$. We must be careful concerning the converse. If there exists $\mathscr{D} \in \beta(\mathfrak{m}) \backslash \mathfrak{m}$ with $x=\mathscr{D}-\lim _{\xi<\mathfrak{m}} x_{\xi}$, then $x \in\left\{x_{\xi}: \xi<\mathfrak{m}\right\}^{\prime}$, but $x$ is not necessarily a cluster point of the net $\left(x_{\xi}: \xi<\mathfrak{m}\right)$, for example: if $\left(x_{n}: n \in N\right)$ converges to $x$ but $x \notin\left\{x_{\xi}: \omega_{0} \leqslant \xi<\omega_{1}\right\}^{\prime}$, then for any $\mathscr{D} \in \beta\left(\omega_{0}\right) \backslash \omega_{0}$, $x=\mathscr{D}-\lim _{\xi<\omega_{1}} x_{\xi}$ but $x$ is not a cluster point of the net $\left(x_{\xi}: \xi<\omega_{1}\right)$.

(iv) A sequence $\left(x_{n}: n \in N\right)$ converges to $x$ if, and only if, $x=\mathscr{D}-$ $\lim _{n \rightarrow \infty} x_{n}$, for each $\mathscr{D} \in \beta N \backslash N$.

(v) If a transfinite sequence $\left(x_{\xi}: \xi<\mathfrak{m}\right)$ converges to $x$, then $x=\mathscr{D}-$ $\lim _{\xi<m} x_{\xi}$, for each $\mathscr{D} \in \mu \mathrm{m}$. Conversely if $\mathfrak{m}$ is regular, then if $x=\mathscr{D}-$ $\lim _{\xi<\mathfrak{m}} x_{\xi}$ for each $\mathscr{D} \in \mu \mathrm{m}$, then $\left(x_{\xi}: \xi<\mathfrak{m}\right)$ converges to $x$. If $\mathfrak{m}$ is singular, then if $x=\mathscr{D}-\lim _{\xi<m} x_{\xi}$ for each $\mathscr{D}$ such that $\mathscr{D} \in \mu \mathrm{n}$ and $\operatorname{cf}(\mathrm{m}) \leqslant \mathfrak{n} \leqslant$ $\mathfrak{m}$, then $\left(x_{\xi}: \xi<\mathfrak{m}\right)$ converges to $x$. (Here as usual $\mathrm{cf}(\mathfrak{m})$ denotes the smallest cardinal cofinal in $\mathrm{m}$.)

(vi) Let $f: X \rightarrow Y$ be a continuous map, $\left(x_{\xi}: \xi<m\right)$ a net in $X$, and let $z \in X$ with $z=\mathscr{D}-\lim _{\xi<m} x_{\xi}$. Then

$$
f(z)=\mathscr{D}-\lim _{\xi<m} f\left(x_{\xi}\right)
$$

2. Product spaces. The usefulness and elegance of the 10 -limit concept is illustrated by the following results. We need some definitions.

A space $X$ is $[\mathfrak{n}, \mathfrak{m}]$-compact if every open cover of $X$ of cardinality $\leqslant \mathfrak{m}$ has a subcover of cardinality $<\mathfrak{n}$.

A space $X$ has property $C[\mathfrak{n}, \mathfrak{m}]$ provided that if $M \subset X$ and $\mathfrak{n} \leqslant|M| \leqslant$ $\mathfrak{m}$, then $M$ has a complete accumulation point in $X$.

The definition $C[n, m]$ is made in the spirit of $\left[V_{1}\right]$, and we will show that this property is different from any of the properties discussed in $\left[\mathbf{V}_{1}\right]$. This property was formally introduced by $\mathrm{Yu}$. M. Smirnov in $\left[\mathrm{S}_{3}\right]$ under the name " $A^{0}$ in the interval $[\mathfrak{n}, \mathfrak{m}]$ ". In [CS], the property $C[\mathfrak{n}, \mathrm{m}]$ is called " $\left[\mathfrak{n}, \mathrm{m}^{+}\right]-$ compact in the sense of complete accumulation points". The property $\left[\aleph_{0}, \mathfrak{m}\right]$-compact is sometimes called initially $m$-compact $[\mathbf{S V}],\left[\mathbf{V}_{2}\right]$. It is not difficult to show that a space is $\left[\kappa_{0}, \mathfrak{m}\right]$-compact if, and only if, it has $C\left[\kappa_{0}, \mathfrak{m}\right]$. Additional results concerning the property $C[\mathrm{n}, \mathrm{m}]$ will come in $\$ 6$.

Many of our results concerning product spaces depend on the following pleasant fact, whose proof is trivial.

THEOREM 2.1. If $X=\Pi_{i \in I} X_{i}$, and for each $i \in I$, we have $x_{i} \in X_{i}$ and ( $x_{\xi}^{i}$ : $\xi<\mathfrak{m}) a$ net in $X_{i}$ with $x_{i}=\mathscr{D}-\lim _{\xi<\mathfrak{m}} x_{\xi}^{i}$, then with $x=\left(x_{i}: i \in I\right)$ and $x_{\xi}=\left(x_{\xi}^{i}: i \in I\right)$, then we have 


$$
x=\text { OD }-\lim _{\xi<m} x_{\xi} \text {. }
$$

Lemma 2.2. Let $X=\prod_{i \in I} X_{i}$. Then $X$ is $C[\mathfrak{n}, \mathfrak{m}]$ if, and only if, for every a such that $\mathfrak{n} \leqslant a \leqslant \mathfrak{m}$, and every net $\left(x_{\xi}: \xi<a\right)$ with $x_{\xi}=\left(x_{\xi}^{i}: i \in I\right)$, and $\left(x_{\xi}\right.$ : $\xi<$ a) faithfully indexed, then there exist $\mathscr{D} \in \mu \mathrm{a}$ and $x_{i} \in X_{i}$ with $x_{i}=\mathscr{D}-$ $\lim _{\xi<a} x_{\xi}^{i}$, i.e., if $x=\left(x_{i}: i \in I\right)$, then $x=\mathscr{D}-\lim _{\xi<a} x_{\xi}$.

Proof. Suppose that $X$ is $C[\mathfrak{n}, \mathfrak{m}]$. Then the set $\left\{x_{\xi}: \xi<\mathfrak{a}\right\}$ has a complete accumulation point $x$ in $X$ and hence there exists $\mathscr{D} \in \mu \mathrm{a}$ with $x=\mathscr{D}$ $\lim _{\xi<a} x_{\xi}$.

Conversely, if $\left\{x_{\xi}: \xi<\mathfrak{a}\right\}$ is a set of distinct points of $X$ with $\mathfrak{n} \leqslant \mathfrak{a} \leqslant \mathfrak{m}$, then there exist $x \in X$ and $\mathscr{D} \in \mu \mathrm{a}$ such that $x=\mathscr{D}-\lim _{\xi<\mathrm{a}} x_{\xi}$, and so $x$ is a complete accumulation point of $\left\{x_{\xi}: \xi<a\right\}$.

Theorem 2.3. Let $X=\prod_{i \in I} X_{i}$. Then $X$ is $C[\mathfrak{n}, \mathfrak{m}]$ if and only if $\prod_{i \in J} X_{i}$ is $C[\mathfrak{n}, \mathfrak{m}]$, for each $J \subset I$ such that $|J| \leqslant 2^{2^{\mathrm{m}}}$.

Proof. If $X$ is not $C[\mathfrak{n}, \mathfrak{m}]$, then there exists $M \subset X,|M|=\mathfrak{a}$ with $\mathfrak{n} \leqslant \mathfrak{a} \leqslant \mathfrak{m}$ and $M$ has no complete accumulation point in $X$. Set $M=\left\{x_{\xi}\right.$ : $\xi<\mathfrak{a}\}$, and each $x_{\xi}=\left(x_{\xi}^{i}: i \in I\right)$. By Lemma 2.2, for each $\mathscr{D} \in \mu \mathfrak{a}$, there is $f(\mathscr{D}) \in I$ for which $\left(x_{\xi}^{f(\mathscr{D})}: \xi<a\right)$ has no $\mathscr{D}$-limit point in $X_{f(\mathcal{D})}$. Let $J=$ $f[\mu \mathrm{a}] \cup K$ where $|K| \leqslant a$ and we include $K$ in $J$ to insure that if $\pi$ : $X \rightarrow \prod_{i \in J} X_{i}$ is the natural projection, then $\pi$ is one-to-one on $M$. Note that $|J| \leqslant 2^{2^{\mathrm{a}}} \leqslant 2^{2^{\mathrm{m}}}, \pi(M) \subset \prod_{i \in J} X_{i}$ has no $\mathscr{D}$-limit point, for any $\mathscr{D} \in \mu \mathrm{a}$, and so $\pi(M)$ has no complete accumulation point in $\prod_{i \in J} X_{i}$.

The reverse implication is trivial.

The next result follows as a special case of Theorem 2.3. Recall [AU] that a space is countably compact if and only if it is $C\left[\kappa_{0}, \aleph_{0}\right]$.

THeOREM 2.4. Let $X=\Pi X_{i}$. If every product of at most $2^{c}$ of the spaces $X_{i}$ is countably compact, then $X$ is countably compact.

This improves a theorem in $\left[\mathbf{S S}_{2}\right]$, which says that $X$ is countably compact provided that every product of at most $2^{2^{c}}$ of the spaces $X_{i}$ is countably compact.

It would be nice to show that Theorem 2.4 is a best possible result (that is, that $2^{c}$ cannot be replaced by any smaller cardinal number), and with some additional set-theoretic assumptions, we are able to do so. Our first example is a natural one to consider.

For each $p \in \beta N \backslash N$, let $K_{p}=\beta N \backslash\{p\}$. It is well known, and easy to prove, that $\Pi_{p \in \beta N \backslash N} K_{p}$ is not countably compact. Thus we would like to show that if $J \subset \beta N \backslash N$ and $|J|<2^{c}$, then $\prod_{p \in J} K_{p}$ is countably compact. An early version of this paper showed that if one assumed $\kappa_{1}=c$ and $\kappa_{2}=2^{c}$, then if $|J|<2^{c}$ then $\Pi_{p \in J} K_{p}$ is countably compact. The proof used Theorem 
2.4 of $\left[\mathbf{S S}_{1}\right]$. A recent result of $\mathrm{S}$. $\mathrm{H}$. Hechler in $[\mathbf{H}]$ shows that Martin's Axiom implies that if $|J|=c$, then $\Pi_{p \in J} K_{p}$ is countably compact. Thus Martin's Axiom plus $c^{+}=2^{c}$ are enough to guarantee that if $|J|<2^{c}$, then $\Pi_{p \in J} K_{p}$ is countably compact. It seems possible that still weaker set-theoretic assumptions would be sufficient for this purpose, but to our knowledge, this remains an open question.

We will now show that Martin's Axiom is sufficient to prove that $2^{c}$ is a best possible result in Theorem 2.4. I am grateful to Wis Comfort for the basic idea of the example we will construct, which is similar to a technique he used in [C] to solve a parallel problem concerning pseudocompact product spaces.

To perform the construction we need the concept of a Ramsey ultrafilter.

Definition. An ultrafilter $\mathscr{D}$ on $N$ is a Ramsey ultrafilter if whenever $\left\{A_{n}\right.$ : $n \in N\}$ is a countable decomposition of $N$ and for all $n \in N, A_{n} \notin \mathscr{Q}$, then there exists $A \in \mathcal{D}$ with $\left|A \cap A_{n}\right| \leqslant 1$, for all $n$.

Clearly a Ramsey ultrafilter is a $P$-point, since an ultrafilter $\mathscr{D}$ on $N$ is a $P$-point if whenever $\left\{A_{n}: n \in N\right\}$ is a countable decomposition of $N$ and for all $n \in N, A_{n} \notin \mathscr{D}$, then there exists $A \in \mathscr{D}$ with $\left|A \cap A_{n}\right|<\aleph_{0}$, for all $n$. This can be shown directly, as in Lemma 9.14 of [CN]. In Theorem 9.6 of [CN], a collection of properties equivalent to the Ramsey condition is given. In Theorem 4.12 of $\left[\mathbf{B}_{2}\right]$, D. Booth showed that Martin's Axiom implies that not every $P$-point of $\beta N \backslash N$ is a Ramsey ultrafilter. In [ $\mathbf{B}_{3}$ ], A. Blass has shown that Martin's Axiom implies the existence of $2^{c}$ Ramsey ultrafilters on $N$, and in particular the existence of $2^{c}$ different types of Ramsey ultrafilters on $N$, which is the result we need.

The following notation will be useful: If $\mathscr{D} \in \beta N \backslash N, x=\mathscr{D}-$ $\lim _{n \rightarrow \infty} x_{n}$ and $S \subset N$ with $S \in \mathscr{D}$, then $x=\mathscr{D}-\lim _{n \in S} x_{n}$, indicating that $x$ is a $\mathscr{D}$-limit of the sequence $\left(x_{n}: n \in S\right)$. Clearly if $\mathscr{D}$ is a Ramsey ultrafilter, $x=\mathscr{D}-\lim _{n \rightarrow \infty} x_{n}$ and $\left\{n: x_{n} \neq x\right\} \in \mathscr{D}$, then there exists $S \in$ D with $x=\mathscr{D}-\lim _{n \in S} x_{n}$ and $\left(x_{n}: n \in S\right)$ is faithfully indexed.

We now offer two lemmas which may be of some interest in their own right. I am grateful to John Ginsburg for a conversation in which we worked out the proofs to the lemmas.

LEMMA 2.5. Let $\mathscr{D}$ be a P-point of $\beta N \backslash N, x \in \beta N \backslash N,\left(x_{n}: n \in N\right) a$ faithfully indexed sequence in $\beta N \backslash N$, and $x=\mathscr{D}-\lim _{n \rightarrow \infty} x_{n}$. Then there exists $A \in \mathscr{D}$ with $x=\mathscr{D}-\lim _{n \in A} x_{n}$ and $\left\{x_{n}: n \in A\right\}$ is discrete.

Proof. Since $\left(x_{n}: n \in N\right)$ is faithfully indexed, we can clearly assume without loss of generality that $x_{n} \neq x$, for all $n$. Hence no $x_{n}$ is a $\mathscr{D}$-limit point of $\left(x_{n}: n \in N\right)$. So for each $n$, there exists $M_{n} \subset N$ with $M_{n} \in x_{n}$ and $T_{n}=\left\{k \in N: M_{n} \in x_{k}\right\} \notin \mathscr{D}$. Since $\mathscr{D}$ is a $P$-point there exists $A \in \mathscr{D}$ with 
$\left|A \cap T_{n}\right|<\aleph_{0}$, for all $n$. Then $x=\mathscr{D}-\lim _{n \in A} x_{n}$, since $A \in \mathscr{D}$. Moreover $M_{n} \in x_{n}$ and $\left\{k \in A: M_{n} \in x_{k}\right\}=T_{n} \cap A$ is finite. Thus the set $\left\{x_{n}\right.$ : $n \in A\}$ is discrete.

LEMMA 2.6. Let $\mathscr{D}_{1}$ and $\mathscr{D}_{2}$ be P-points of $\beta N \backslash N$ of distinct types. Let $x$ and $y$ be points of $\beta N \backslash N,\left(x_{n}: n \in N\right)$ and $\left(y_{n}: n \in N\right)$ faithfully indexed sequences in $\beta N, x=\mathscr{D}_{1}-\lim _{n \rightarrow \infty} x_{n}$ and $y=\mathscr{D}_{2}-\lim y_{n}$. Then $x \neq y$.

Proof. Lemma 2.5 guarantees that we can assume that the sets $\left\{x_{n}\right.$ : $n \in N\}$ and $\left\{y_{n}: n \in N\right\}$ are discrete. We also assume, as in the previous proof, that for all $n, x_{n} \neq x, x_{n} \neq y, y_{n} \neq x$, and $y_{n} \neq y$. Then as before, for each $k \in N, y_{k} \neq \mathscr{D}_{1}-\lim _{n \rightarrow \infty} x_{n}$ so there exists $M_{k} \in y_{k}$ for which $T_{k}=$ $\left\{n: M_{k} \in x_{n}\right\} \notin \mathscr{D}_{1}$. Then there exists $A \in \mathscr{D}_{1}$ with $\left|A \cap T_{k}\right|<\aleph_{0}$, for all $k$. Then $x=\mathscr{D}_{1}-\lim _{n \in A} x_{n}$ and for each $k, y_{k} \notin\left\{x_{n}: n \in A\right\}^{\prime}$.

Similarly find $B \in \mathscr{D}_{2}$ with $y=\mathscr{D}_{2}-\lim _{n \in B} y_{n}$ and for each $k, x_{k} \notin\left\{y_{n}\right.$ : $n \in B\}^{\prime}$. Then $\left\{x_{n}: n \in A\right\} \cup\left\{y_{n}: n \in B\right\}$ is discrete.

Set $Z=\left\{x_{n}: n \in A\right\} \cup\left\{y_{n}: n \in B\right\}$. Then $\operatorname{cl} Z \approx \beta N$ and $Z^{\prime}=\operatorname{cl} Z \backslash Z$ $\approx \beta N \backslash N$.

Now we will apply the theory of relative types, i.e., a point $p$ in $Z^{\prime}$ has a type as a point of $\mathrm{cl} Z$ as well as a type as a point of $\beta N\left[\mathbf{F}_{1}, 1.5\right]$, [SS $\mathbf{S S}_{3}$ Lemma 1.1] or [W, Lemma 3.42] and [SS $]$, [W] if $W \subset Z$ and $p \in W^{\prime}$, then the type of $p$ with respect to $W$ is the same as the type of $p$ with respect to $Z$.

Thus in our example, the type of $x$ with respect to $Z$ is the type of $\mathscr{D}_{1}$, and the type of $y$ with respect to $Z$ is the type of $\mathscr{D}_{2}$. Since $\mathscr{D}_{1}$ and $\mathscr{D}_{2}$ have different types, $x \neq y$.

We are now ready to construct the promised example. Note that the hypothesis of Ramsey ultrafilter (as opposed to $P$-point) is used only in the first line of the proof, where, in order to apply Lemma 2.6, we need to assume that the sequences can be chosen to be faithfully indexed.

THEOREM 2.7. Let I be the set of types of Ramsey ultrafilters on $N$ and select $\mathscr{D}_{i}$ of type $i$, for each $i$.

Set $F_{i}=\left\{x \in \beta N \backslash N\right.$ : there exists a sequence $\left(x_{n}: n \in N\right)$ in $\beta N$ with $x=\mathscr{D}_{i}-\lim _{n \rightarrow \infty} x_{n}$ and $\left.\left\{n: x_{n} \neq x\right\} \in \mathscr{D}_{i}\right\}$.

Set $X_{i}=N \cup \cup_{j \neq i} F_{j}$ and $X=\Pi_{i \in I} X_{i}$. Then $X$ is not countably compact and if $J \subsetneq I$, then $\Pi_{i \in J} X_{i}$ is countably compact. In particular, if $|J|<|I|$, then $\Pi_{i \in J} X_{i}$ is countably compact.

Proof. First, $i \neq j$ implies that $F_{i} \cap F_{j}=\varnothing$, which follows immediately from Lemma 2.6. Then $N$ is (homeomorphic to) the diagonal of $X$ and so $X$ is not countably compact. Now let $J \subsetneq I$ and so there exists $i_{0} \in I \backslash J$. To show that $\Pi_{i \in J} X_{i}$ is countably compact, let $\left(x_{n}: n \in N\right)$ be a sequence in 
$\Pi_{i \in J} X_{i}$ and we need to define a cluster point $x=\left(x_{i}: i \in J\right)$ of the sequence. So take $i \in J$ and consider $\left(\pi_{i}\left(x_{n}\right): n \in N\right)$. If there exists $x \in \beta N$ such that $\left\{n: \pi_{i}\left(x_{n}\right)=x\right\} \in \mathscr{D}_{i_{0}}$, then clearly such $x$ is unique and an element of $X_{i}$ and so we can set $x_{i}=x$. If no such $x$ exists, then the sequence $\left(\pi_{i}\left(x_{n}\right)\right.$ : $n \in N)$ has a unique $\mathscr{D}_{i_{0}}$-limit point $x_{i} \in F_{i_{0}} \subset X_{i}$. Let $x=\left(x_{i}: i \in J\right)$ and since for each $i, x_{i}=\mathscr{D}_{i_{0}}-\lim _{n \rightarrow \infty} \pi_{i}\left(x_{n}\right)$, applying Theorem 2.1 we have $x=\mathscr{D}_{i_{0}}-\lim _{n \rightarrow \infty} x_{n}$. Thus $\Pi_{i \in J} X_{i}$ is countably compact.

Using the previously quoted result of Blass, we can state the relationship of this example to Theorem 2.4 as follows.

THEOREM 2.8. Martin's Axiom implies the existence of a set I of cardinality $2^{c}$ and a family of spaces $\left(X_{i}: i \in I\right)$ such that $\Pi_{i \in I} X_{i}$ is not countably compact and if $J \subset I$ with $|J|<|I|$, then $\Pi_{i \in J} X_{i}$ is countably compact.

Note that for any $m \leqslant 2^{c}$, this theorem holds with $m$ in place of $2^{c}$.

We will now offer a new proof of the Tychonoff theorem.

Defintion. Let $\mathscr{D} \in \mu \mathrm{m}$. A space $X$ is $\mathscr{D}-m$-compact provided that each transfinite sequence $\left(x_{\xi}: \xi<\mathfrak{m}\right)$ has a $\mathscr{D}$-limit point in $X$.

THEOREM 2.9. The following assertions are equivalent for $X$.

(a) $X$ is compact;

(b) $X$ is $\mathscr{D}-\mathfrak{m}$-compact, for all $\mathfrak{m}$ and for all $\mathscr{D} \in \mu \mathfrak{m}$;

(c) $X$ is $\mathscr{D}-\mathfrak{m}$-compact, for all $\mathfrak{m} \leqslant|X|$ and for all $\mathscr{D} \in \mu \mathrm{m}$.

Proof. (a) $\Rightarrow(b)$ Let $\left(x_{\xi}: \xi<\mathfrak{m}\right)$ be a net in $X$ and $\mathscr{D} \in \mu \mathfrak{m}$. We will produce a $\mathscr{D}$-limit point for $\left(x_{\xi}: \xi<\mathfrak{m}\right)$ in $X$. For each $K \in \mathbb{D}$, set $F_{K}=$ $\left\{x_{\xi}: \xi \in K\right\}$. Then

$$
F_{K_{1}} \cap \cdots \cap F_{K_{n}}=F_{K_{1} \cap \cdots \cap K_{n}}
$$

and so $\left(F_{K}: K \in \mathscr{D}\right)$ has the finite intersection property. Since $X$ is compact, there exists $p \in \cap_{K \in \mathscr{D}} \bar{F}_{K}$. Take $p \in U$ open and set $M=\left\{\xi: x_{\xi} \in U\right\}$. Then since for each $K \in \mathscr{D}, U \cap F_{K} \neq \varnothing$, we have, for each $K \in \mathscr{D}$, $M \cap K \neq \varnothing$, i.e., $M \in \mathscr{D}$, so $p=\mathscr{D}-\lim _{\xi<m} x$.

(b) $\Rightarrow$ (c) is trivial.

(c) $\Rightarrow$ (a) This is easy, since if $A \subset X$ is infinite, then $|A|=\mathfrak{m} \leqslant|X|$ and set $A=\left(a_{\xi}: \xi<\mathfrak{m}\right)$. Take $\mathscr{D} \in \mu \mathfrak{m}$, and there exists $a \in X$ with $a=\mathscr{D}-$ $\lim _{\xi<m} a_{\xi}$. Thus $A$ has a complete accumulation point in $X$, and so $X$ is compact.

This result can be contrasted with a later result, where we show that if there exists $p \in \beta N \backslash N$ such that $p$ is not an accumulation point of any countable subset of $\beta N \backslash N$, then there exists a Hausdorff space $X$ which is $\mathscr{D}-\kappa_{0}$ compact for every $\mathscr{D} \in \beta N \backslash N$ but $X$ is not $\kappa_{0}$-bounded.

Since it is immediate that every product of $\mathscr{D}-m$-compact spaces is 
D $-m$-compact, we have a proof that the product of compact spaces is compact $\left[\mathbf{S}_{2}\right]$.

3. A space is characterized by its $\mathscr{D}$-limits. In this section we exhibit several properties possessed by the collection of $\mathscr{D}$-limits of a space. It is also shown that in any space, the topology is determined by the $D$-limits of the space.

The following result is essential to the remainder of this paper.

THEOREM 3.1. Let $X$ be a space, $A=\left\{a_{\gamma}: \gamma<\mathfrak{n}\right\}$ dense in $X, x=\mathscr{D}-$ $\lim _{\xi<m} x_{\xi}$, each $x_{\xi}=\mathscr{D}_{\xi}-\lim _{\gamma<\mathfrak{n}} a_{\gamma}$, and suppose that in $\beta(\mathfrak{n}), y=\mathscr{D}-$ $\lim _{\xi<m} D_{\xi}$. Then in $X, x=y-\lim _{\gamma<n} a_{\gamma}$.

Proof. Let $x \in V$ open and set $L=\left\{\gamma: a_{\gamma} \in V\right\}$. We need to show that $L \in y$.

Set $K=\left\{\xi: x_{\xi} \in V\right\}$. Then $K \in \mathscr{D}$ and so $y \in \operatorname{cl}\left\{\mathscr{D}_{\xi}: \xi \in K\right\}$. For each $\xi \in K, L \in \mathscr{D}_{\xi}$.

Thus $y \in \operatorname{cl}\left\{\mathscr{D}_{\xi}: \xi \in K\right\} \subset \bar{L}$ and so $L \in y$.

This fact is very natural; it says essentially that if a sum of ultrafilters can be taken in $\beta(\mathfrak{n})$, then if we take the same sum of ultrafilters in a space $X$, the answer in $X$ will reflect the answer in $\beta(\mathfrak{n})$. We will use this fact in several constructions.

We will now show that any space is characterized by its $\mathscr{D}$-limits, and conversely, we exhibit necessary and sufficient conditions that a specified collection of $\mathscr{D}$-limits on a set $X$ yield a topology. The conditions are natural, i.e., that sums in $\beta(\mathfrak{n})$ be respected and that the property of an ultrafilter being closed under the taking of finite intersections be respected.

THEOREM 3.2. Let $X$ be a set with $X=\mathfrak{m}$. For each $\mathscr{D} \in \beta(\mathfrak{m})$, suppose that we have a collection $C(D)$ of ordered pairs, where the first element of the pair is a net, indexed by $\mathfrak{n}$, of points of $X$ where $\mathscr{D} \in \beta(\mathfrak{n}) \subset \beta(\mathfrak{m})$, and the second element of the pair is a point of $X$. For each $A \subset X$ set

$$
\bar{A}=A \cup\left\{x \in X: \exists \mathscr{D} \in \beta(\mathfrak{m}) \backslash \mathfrak{m} \exists\left(x_{\xi}: \xi<\mathfrak{n}\right) \subset A\right.
$$

such that $\left.\left(\left(x_{\xi}: \xi<\mathfrak{n}\right), x\right) \in \mathcal{C}(\mathscr{D})\right\}$.

The following are equivalent:

(a) "-" is a closure operator and $\left(\left(x_{\xi}: \xi<\mathfrak{n}\right), x\right) \in \mathcal{C}(\mathcal{D})$ if and only if $x=\mathscr{D}-\lim _{\xi<n} x_{\xi}$ in the topology induced on $X$ by ${ }^{-}$.

(b) the collections $\mathrm{C}(\mathbb{D})$ satisfy the following two conditions:

(i) If $x,\left(x_{\xi}: \xi<\mathfrak{t}\right)$, and $\left(a_{\gamma}: \gamma<\mathfrak{n}\right)$ are elements of $X$ and $\left(\left(x_{\xi}: \xi<\mathfrak{t}\right)\right.$, $x) \in \mathcal{C}(\mathscr{D}),\left(\left(a_{\gamma}: \gamma<\mathfrak{n}\right), x_{\xi}\right) \in \mathcal{C}\left(\mathscr{D}_{\xi}\right)$, and if in $\beta(\mathfrak{n}), y=\mathscr{D}-\lim _{\xi<t} \mathscr{D}_{\xi}$, then $\left(\left(a_{\gamma}: \gamma<\mathfrak{n}\right), x\right) \in \mathcal{C}(y)$.

(ii) If $\left(\left(x_{\xi}: \xi<\mathfrak{n}\right), x\right) \in \mathcal{C}(\mathbb{D})$ and if $\left(y_{\xi}: \xi<\mathfrak{t}\right)$ is such that $\left\{\xi: x_{\xi}=y_{\xi}\right\} \in$ D, then $\left(\left(y_{\xi}: \xi<\mathfrak{t}\right), x\right) \in \mathcal{C}(\mathscr{D})$. Here $\omega \leqslant \mathfrak{t} \leqslant \mathfrak{n}$. 
Moreover, if "-" is a closure operator, then $X$ is $T_{1}$ if, and only if, (iii) whenever $\left\{\xi: x_{\xi}=x\right\} \in \mathscr{D}$ and $y \neq x$, then $y \neq \mathscr{D}-\lim _{\xi<\mathfrak{n}} x_{\xi}$.

Furthermore, a $T_{1}$ space is Hausdorff if, and only if

(iv) $x=\mathscr{D}-\lim _{\xi<\mathfrak{n}} x_{\xi}$ and $y=\mathscr{D}-\lim _{\xi<\mathrm{t}} x_{\xi}$ implies that $x=y$.

Proof. If $X$ is a topological space, then (i) is Theorem 3.1 and (ii) is obvious.

To show the converse it is trivial that $\bar{\phi}=\phi$ and that $A \subset \bar{A}$ for all $A \subset X$. The condition $\bar{A} \subset \bar{A}$ is implied directly by condition (i) so it remains to show that $\overline{A \cup B}=\bar{A} \cup \bar{B}$, for all $A, B \subset X$. It is immediate if $A \subset B$, then $\bar{A} \subset \bar{B}$ so we need to show $\overline{A \cup B} \subset \bar{A} \cup \bar{B}$, i.e., if $x=\mathscr{D}-\lim _{\xi<n} x_{\xi}$ with $\left\{x_{\xi}: \xi<\mathfrak{n}\right\} \subset A \cup B$ and $x \notin A \cup B$, then $x \in \bar{A} \cup \bar{B}$. We assume that $A \backslash B$ and $B \backslash A$ are both nonempty, so take $a \in A \backslash B$ and $b \in B \backslash A$.

$$
\begin{array}{cc}
\text { Define } y_{\xi}=x_{\xi} \text { if } x_{\xi} \in A & \text { Define } z_{\xi}=x_{\xi} \text { if } x_{\xi} \in B \\
\text { and } y_{\xi}=a \text { if } x_{\xi} \notin A . & \text { and } z_{\xi}=b \text { if } x_{\xi} \notin B . \\
\text { If }\left\{\xi: y_{\xi}=x_{\xi}\right\} \in \mathscr{D}, \text { then } & \text { If }\left\{\xi: z_{\xi}=x_{\xi}\right\} \in \mathscr{D}, \text { then } \\
x=\mathscr{D}-\lim _{\xi<\mathfrak{n}} y_{\xi} \text { and } x \in \bar{A} . & x=\mathscr{D}-\lim _{\xi<\mathfrak{n}} z_{\xi} \text { and } x \in \bar{B} .
\end{array}
$$

If both fail, then $\phi=\left\{\xi: x_{\xi} \notin A\right\} \cap\left\{\xi: x_{\xi} \notin B\right\} \in \mathscr{D}$, which is a contradiction.

The $T_{1}$ condition requires no comment. See $\left[\mathbf{M}_{2}\right]$.

If $X$ is Hausdorff, then it is easy to see that condition (iv) is satisfied, because one of $\mathfrak{n}$ and $t$ is greater than or equal to the other, say $\mathfrak{n} \leqslant t$, and restricting the $t$-net to $\mathfrak{n}$, the result follows from the uniqueness of the $\mathscr{D}$-limit of a fixed net.

We now show that a space with unique $\mathscr{D}$-limits is Hausdorff. First, such a space is $T_{1}$. To verify condition (iii), suppose that $\left\{\xi: x_{\xi}=x\right\} \in \mathscr{D}$ and $y \neq x$. Since $x=\mathscr{D}-\lim _{\xi<n} x_{\xi}$, condition (iv) says that $y \neq \mathscr{D}-$ $\lim _{\xi<n} x_{\xi}$.

Now suppose that the space is not Hausdorff, i.e., there exist $x, y \in X$ with $x \neq y$ and whenever $x \in U$ open and $y \in V$ open then $U \cap V \neq \varnothing$. So take $x_{(U, V)} \in U \cap V$. Well order (according to a cardinal) $\left\{x_{(U, V)}: U\right.$ and $V$ open, $x \in U, y \in V\}$ so we write this set as the net $\left(x_{\xi}: \xi<\mathfrak{m}\right)$. We will show that there exists $\mathscr{D} \in \beta(\mathfrak{m}) \backslash \mathfrak{m}$ with $x=\mathscr{D}-\lim _{\xi<\mathfrak{m}} x_{\xi}$ and $y=\mathscr{D}-$ $\lim _{\xi<m} x_{\xi}$, which is the desired contradiction. Clearly we have $x \in\left\{x_{\xi}\right.$ : $\xi<\mathfrak{m}\}^{\prime}$ and $y \in\left\{x_{\xi}: \xi<\mathfrak{m}\right\}^{\prime}$. Now take $s(U, V)=\left\{\xi: x_{\xi} \in U \cap V\right\}$. We will show that $s(U, V)$ is infinite. If either $x$ or $y$ is in $U \cap V$, then $s(U, V)$ is infinite, since the space is $T_{1}$. If neither $x$ nor $y$ is in $U \cap V$, let $U=U_{1}$ and there exists $x_{1}=x_{\left(U_{1}, V\right)} \in U \cap V$. Note that $x \neq x_{1}$. Now inductively define $U_{n}=U_{n-1} \backslash\left\{x_{n-1}\right\}$ and there exists $x_{n}=x_{\left(U_{n}, V\right)} \in U_{n} \cap V \subset U \cap V$. 
Clearly $\left\{x_{n}: n \in N\right\}$ is an infinite subset of $s(U, V)$. Now take

$$
\mathcal{G}=\{s(U, V) \backslash\{\xi\}: x \in U \text { open, } y \in V \text { open, } \xi<\mathfrak{m}\} .
$$

Then $\mathcal{G}$ has the finite intersection property so $\mathcal{G} \subset \mathscr{D}$, for some $\mathscr{D} \in \beta(\mathfrak{m}) \backslash$ m. Clearly $x=\mathscr{D}-\lim _{\xi<m} x_{\xi}$ and $y=\mathscr{D}-\lim _{\xi<\mathfrak{m}} x_{\xi}$.

\section{Perfect maps.}

DefintTons. Let $f: X \rightarrow Y$ be an onto function. Then

(i) $f$ is perfect if $f$ is a closed map and $f^{-1}(y)$ is compact for each $y \in Y$. Note that $f$ is not necessarily continuous.

(ii) $f$ is $\mathscr{D}$-perfect if whenever $y=\mathscr{D}-\lim _{\xi<m} f\left(x_{\xi}\right)$ then there exists $x \in X$ such that $x=\mathscr{D}-\lim _{\xi<m} x_{\xi}$ and $f(x)=y$.

(iii) $f$ is $\mathscr{D}$-continuous if whenever $x=\mathscr{D}-\lim x_{\xi}$, then $f(x)=\mathscr{D}-$ $\lim f\left(x_{\xi}\right)$.

(iv) A subset $A$ of $X$ is $\mathscr{D}$-closed in $X$ if whenever $x \in X, x=\mathscr{D}-\lim x_{\xi}$, $\left\{x_{\xi}: \xi<\mathfrak{m}\right\} \subset A$, then $x \in A$.

(v) $f$ is $\mathscr{D}$-closed if $A \mathscr{D}$-closed in $X$ implies that $f[A]$ is $\mathscr{D}$-closed in $Y$.

Easily checked facts are:

(a) $f$ is continuous if, and only if, $f$ is $\mathscr{D}$-continuous, for every $\mathscr{D} \in \beta(\mathfrak{m})$, for every $\mathrm{m}$.

(b) $A$ is closed if, and only if, $A$ is $\mathscr{D}$-closed, for every $\mathscr{D} \in \beta(\mathfrak{m})$, for every m.

(c) If $f$ is $\mathscr{D}$-closed for every $\mathscr{Q}$, then $f$ is closed.

(d) The converse to (c) is false. Let $\mathscr{D} \in \beta N \backslash N, T \subset \beta N \backslash N$ be all ultrafilters of type $\mathscr{D}$. Let $X=\beta N \backslash T$, and $Y=N \cup\{*\}$ be the one-point compactification of $N$. Define $f: X \rightarrow Y$ by $f(n)=n$ for all $n \in N$, and $f(x)=*$ for all $x \in X \backslash N$. Then $f$ is closed but not $\mathscr{D}$-closed.

(e) If $X$ is $\mathscr{D}$ - m-compact and $A$ is closed in $X$ or if $A$ is $D$-closed in $X$, then $A$ is $\mathscr{D}$ - m-compact.

(f) If $f$ is $\mathscr{D}$-perfect, then $f$ is $\mathscr{D}$-closed.

(g) The converse to (f) is false. Let $Y=\{y\}$ be a singleton space and define $f: N \rightarrow Y$ by $f(n)=y$, for all $n \in N$. Then $f$ is $\mathscr{D}$-closed for every $\mathscr{D}$ but $f$ is not $\mathscr{D}$-perfect for any $\mathscr{D} \in \beta N \backslash N$.

(h) If $X$ is $\mathscr{D}-$ m-compact, $Y$ is Hausdorff, and $f$ is continuous, then $f$ is (D)-perfect.

ProOF OF (h). Let $y=\mathscr{D}-\lim _{\xi<\mathfrak{m}} f\left(x_{\xi}\right)$. Consider $\left(x_{\xi}: \xi<\mathfrak{m}\right)$ in $X$ and since $X$ is $\mathscr{D}-\mathfrak{m}$-compact, there exists $x \in X$ such that $x=\mathscr{D}-\lim x_{\xi}$. Since $f$ is continuous, $f(x)=\mathscr{D}-\lim f\left(x_{\xi}\right)$. Since $Y$ is Hausdorff, $\mathscr{D}$-limits are unique, and so $f(x)=y$.

THeOREM 4.1. Let $Y$ be Hausdorff and let $f: X \rightarrow Y$ be continuous onto. Then $f$ is perfect if and only if $f$ is $\mathscr{D}$-perfect for every $\mathscr{D}$. 
Proof. Suppose that $f$ is perfect and we assume, for the sake of a contradiction, that there exists $\mathscr{D}$ for which $f$ is not $\mathscr{D}$-perfect. Then in $Y$ there exist $y=\mathscr{D}-\lim _{\xi<\mathfrak{m}} y_{\xi}, y_{\xi}=f\left(x_{\xi}\right)$, and $\left(x_{\xi}: \xi<\mathfrak{m}\right)$ has no $\mathscr{D}$-limit point in $X$. This is so because if there were $x \in X$ with $x=\mathscr{D}-\lim x_{\xi}$, then as in the proof of $(\mathrm{h})$ we would have $f(x)=y$. Then for each $x \in f^{-1}(y)$, there exists $U_{x}$ open, $x \in U_{x}$, and $\left\{\xi: x_{\xi} \in U_{x}\right\} \notin \mathscr{Q}$. Since $f^{-1}(y)$ is compact and $f^{-1}(y) \subset \cup_{x \in f^{-1}(y)} U_{x}$, we can find a finite subcover $f^{-1}(y) \subset$ $\cup{ }_{i=1}^{n} U_{x_{i}}$. Let $U=\cup_{i=1}^{n} U_{x_{i}}$ and so $f^{-1}(y) \subset U$ and $\left\{\xi: x_{\xi} \in U\right\} \notin \mathscr{D}$. Consider $\left\{\xi: x_{\xi} \notin U\right\}=M \in \mathscr{D}$. Take $B=\left\{x_{\xi}: \xi \in M\right\}$, and $U \cap B=\varnothing$. Since $f$ is closed, $\overline{f[\underline{B}]} \subset f[\bar{B}]$ is closed in $Y[\mathbf{D}$, p. 87], and $y \in \overline{f[B]} \subset f[\bar{B}]$. So there exists $z \in B$ such that $f(z)=y$. But $z \in U$ open and $U \cap B=\varnothing$ so $z \notin \bar{B}$, and we have a contradiction.

Conversely let $f$ be $\mathscr{D}$-perfect for every $\mathscr{D}$. That $f$ is closed follows immediately from easily checked facts (c) and (f).

To show that $f^{-1}(y)$ is compact for each $y \in Y$, it suffices, by Theorem 2.9, to show that $f^{-1}(y)$ is $\mathscr{D}-m$-compact, for all $\mathfrak{m}$ and all $\mathscr{D}$ with DD $\in \mu \mathrm{m}$. So let $\left(x_{\xi}: \xi<\mathfrak{m}\right)$ be a net in $X$ with $f\left(x_{\xi}\right)=y$, for all $\xi<\mathfrak{m}$. Set $y=\mathscr{D}-\lim _{\xi<\mathfrak{m}} f\left(x_{\xi}\right)$. Then there exists $x \in X$ with $f(x)=y$ and $x=\mathscr{D}-$ $\lim _{\xi<m} x_{\xi}$ and so $\left(x_{\xi}: \xi<m\right)$ has a $\mathscr{D}$-limit point in $X$.

Note that in the converse direction we did not use either the hypothesis that $Y$ is Hausdorff or that $f$ is continuous; thus we have the following result.

THEOREM 4.2. Let $f: X \rightarrow Y$ be an onto function. Then if $f$ is $\mathscr{D}$-perfect for each $\mathcal{D}$, then $f$ is perfect.

In the next section, we will apply Theorem 4.2 in a context in which $f$ is not necessarily continuous.

In [E, p. 167] a result of Henriksen and Isbell in [HI] is quoted:

THEOREM (HENRIKSEN-ISBELL). If $X$ and $Y$ are completely regular Hausdorff spaces and $f: X \rightarrow Y$ is continuous onto, then $f$ is perfect if, and only if, $f^{\beta}(\beta X \backslash X)=\beta Y \backslash Y$.

If $X$ and $Y$ are completely regular Hausdorff spaces and $f: X \rightarrow Y$ is continuous onto, then the equivalence of the conditions $f^{\beta}(\beta X \backslash X)=\beta Y \backslash$ $Y$ and $f$ is $\mathscr{D}$-perfect for every $\mathscr{D}$ is virtually trivial, and the two conditions say pretty much the same thing. Thus Theorem 4.1 can be considered to be a substantial generalization of the theorem of Henriksen and Isbell.

Since it is easy to see that a product of $\mathscr{D}$-perfect maps is $\mathscr{D}$-perfect, then we have

THEOREM $4.3\left[\mathbf{B}_{\mathbf{4}}\right],\left[\mathbf{F}_{2}\right]$. The product of perfect continuous maps is perfect.

In [ $\mathbf{B}_{\mathbf{4}}$, p. 117], it is shown that Theorem 4.3 implies the Tychonoff theorem, so this is another proof of the Tychonoff theorem. 
5. Regular spaces. In this section all regular and completely regular spaces are Hausdorff.

Let $X$ be a Hausdorff space, $A$ dense in $X, A=\left(a_{\xi}: \xi<\mathfrak{m}\right)$ faithfully indexed, and so $|A|=\mathrm{m}$. Let

$$
Y=\left\{\mathscr{D} \in \beta(\mathfrak{m}): \text { there exists } x_{\mathscr{D}} \in X \text { with } x_{\mathscr{D}}=\mathscr{D}-\lim _{\xi<\mathfrak{m}} a_{\xi}\right\} .
$$

Since $\mathscr{D}$-limits of a fixed net are unique, if $x_{\mathscr{D}}$ exists, it is unique. Hence we have a natural map $\phi: Y \rightarrow X$ defined by $\phi(\mathscr{D})=x_{\mathscr{D}}$. Note that $\phi$ is well defined and that if $\xi<\mathfrak{m}$, then $\phi(\xi)=a_{\xi}$. We call $\phi$ the special map and $Y$ the special space of $X$ with respect to its dense subspace $A$, writing $Y=$ $S(X, A)$. Note that the ordering on $A$ does not really affect the space $Y$, that is, if $\sigma: \mathfrak{m} \rightarrow \mathfrak{m}$ is a bijection, then the special space $Y$ associated with $X$ and its dense ordered subspace $\left(a_{\xi}: \xi<\mathfrak{m}\right)$ is naturally homeomorphic to the special space $Y_{\sigma}$ associated with $X$ and its dense ordered subspace $\left(a_{\sigma(\xi)}\right.$ : $\xi<\mathfrak{m})$. It is clear that the choice of $A$ may affect $Y$, i.e., if $|A|<|X|$, then $S(X, A)$ is not homeomorphic to $S(X, X)$ since $S(X, X)$ has a dense discrete subspace of cardinality $|X|$ and $S(X, A)$ does not.

Clearly $\phi$ is onto and if $\mathscr{D} \in Y$, then $\phi$ restricted to $\mathfrak{m} \cup\{\mathscr{D}\}$, henceforth written as $\left.\phi\right|_{\mathfrak{m} \cup\{\mathcal{D}\}}$, is continuous. We need to state the folklore lemma: if $f$ : $H \rightarrow F$ is a function, $E$ is dense in $H,\left.f\right|_{E \cup\{h\}}$ is continuous for each $h \in H$, and $F$ is regular, then $f$ is continuous [BD], [GJ, problem $6 \mathrm{H}$ ].

Thus, if $X$ is regular, then $\phi$ is continuous.

We will now prove that $\phi$ is perfect, and that if $\phi$ is continuous, then $X$ is regular. Then will follow some discussion.

(i) $\phi$ is perfect. By Theorem 4.2, it suffices to show that $\phi$ is $\mathscr{D}$-perfect, for every $\mathscr{D}$. So let $x=\mathscr{D}-\lim _{\delta<\mathfrak{n}} \phi\left(y_{\delta}\right)$ and we need to show that there exists $y \in Y, y=\mathscr{D}-\lim _{\delta<n} y_{\delta}$ and $\phi(y)=x$. By the definition of $\phi, \phi\left(y_{\delta}\right)=y_{\delta}$ $-\lim _{\xi<\mathfrak{m}} a_{\xi}$. In $\beta(\mathfrak{m})$, take $y=\mathscr{D}-\lim _{\delta<n} y_{\delta}$. By Theorem 3.1, $x=y-$ $\lim _{\xi<m} a_{\xi}$ and so $y \in Y$ and $\phi(y)=x$.

(ii) If $\phi$ is continuous, then $X$ is regular. This can be shown directly, as in [D, p. 235].

Thus the following converse of the folklore lemma is true:

THEOREM 5.1. Let $X$ be a Hausdorff space such that whenever $Y$ is a space, $f$ : $Y \rightarrow X$ is a function, $Y$ has dense subspace $M$ and $\left.f\right|_{M \cup\{y\}}$ is continuous, for each $y \in Y$, then $f$ is continuous. Then $X$ is regular.

This is known, it was proved by Bourbaki and Dieudonne [BD] in 1939.

In [GJ], it is shown in Corollary 10.14 that for any completely regular space $X$, if $D$ is a discrete space equipotent with a dense subset of $X$, then $X$ is a continuous perfect image of a suitable subspace of $\beta D$. In the following 
theorem, we summarize our results of this section, and generalize [GJ, Corollary 10.14].

THEOREM 5.2. Let $X$ be a Hausdorff space, $A=\left(a_{\xi}: \xi<\mathfrak{m}\right)$ a dense subspace of $X$. Let $Y=\left\{\mathscr{D} \in \beta(m)\right.$ : there exists $x_{\mathscr{D}} \in X$ with $\left.x_{\mathscr{D}}=\mathscr{D}-\lim _{\xi<m} a_{\xi}\right\}$. Let $\phi: Y \rightarrow X$ be the special map. Then $\phi$ is perfect and $\phi$ is continuous if, and only if, $X$ is regular. Thus if $X$ is a Hausdorff space and $D$ is a discrete space equipotent with a dense subspace of $X$, then $X$ is a continuous perfect image of $a$ suitable subspace of $\beta D$ which contains $D$ if and only if $X$ is regular.

Now let $X$ and $Z$ be Hausdorff spaces, $A$ dense in $X$, and $f: X \rightarrow Z$ is continuous onto. Then we have the special spaces $Y_{X}=S(X, A)$ and $Y_{Z}=$ $S(Z, f[A])$, and the following diagram

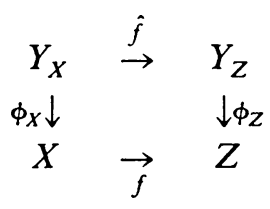

Then there exists a unique $\hat{f}: Y_{X} \rightarrow Y_{Z}$ continuous making the diagram commute. To see this, let $y \in Y_{X}$. Then $y=y-\lim _{\xi<m} \xi$ and there exists $x \in X$ such that $x=y-\lim _{\xi<m} a_{\xi}$. Then

$$
f(x)=y-\lim _{\xi<m} f\left(a_{\xi}\right)
$$

and so there is a unique

$$
\hat{f}(y)=y-\lim _{\xi<\mathfrak{m}} \phi_{Z}^{-1} f\left(a_{\xi}\right) .
$$

Then, for each $y \in Y_{X},\left.\hat{f}\right|_{m \cup\{y\}}$ is continuous, and since $Y_{Z}$ is regular, $\hat{f}$ is continuous. It is now easy to check that if $X$ and $Z$ are regular, then $f$ is perfect if, and only if, $\hat{f}$ is perfect.

After the short communication I gave at the International Congress of Mathematicians in Vancouver, the question was raised as to whether $X$ $\aleph_{0}$-bounded is equivalent to $X$ being ultracompact (defined below). I gave Bernstein's result that $X \aleph_{0}$-bounded implies that $X$ is ultracompact and if $X$ is completely regular ultracompact, then $X$ is $\kappa_{0}$-bounded. Thus the two properties are equivalent when $X$ is completely regular. At that point, I. Juhász reported that the equivalence holds when $X$ is regular, and that his proof is in the context of Non-Standard Analysis (as is most of Bernstein's paper, although his proof that $X$ completely regular ultracompact implies $X$ is $\kappa_{0}$-bounded is topological). We offer a topological proof that $X$ regular ultracompact implies $X$ is $\aleph_{0}$-bounded, as a corollary to a more general result.

First some definitions.

$X$ is $\mathfrak{m}$-bounded if $A \subset X,|A| \leqslant \mathfrak{m}$ imply that $\operatorname{cl}_{X} A$ is compact; 
$X$ is $\mathfrak{m}$-ultracompact if $\mathfrak{n} \leqslant \mathfrak{m}, \mathscr{D} \in \mu \mathfrak{n}$ imply $X$ is $\mathscr{D}-\mathfrak{n}$-compact.

If $X$ is $\aleph_{0}$-ultracompact, then we say that $X$ is ultracompact, as in Bernstein's paper.

The following result is (a) $\Rightarrow$ (c) of Theorem 2.9.

THEOREM 5.3. If $X$ is $\mathfrak{m}$-bounded, then $X$ is m-ultracompact.

THEOREM 5.4. If $X$ is m-ultracompact regular, then $X$ is $\mathfrak{m}$-bounded.

Proof. Let $A \subset X,|A|=\mathfrak{n} \leqslant \mathfrak{m}$. Now take the special space $Y=$ $S\left(\mathrm{cl}_{X} A, A\right)$ and $\phi: Y \rightarrow \mathrm{cl}_{X} A$ the special map. Note that $Y \subset \beta(\mathfrak{n})$. Now write $A=\left(a_{\xi}: \xi<\mathfrak{n}\right)$ and since for each $\mathscr{D} \in \beta(\mathfrak{n})$, there exists $x_{\mathscr{D}}=\mathscr{D}-$ $\lim _{\xi<\mathfrak{m}} a_{\xi}, Y=\beta(\mathfrak{n})$. Since $X$ is regular, $\mathrm{cl}_{X} A$ is regular, so $\phi$ is continuous and $\mathrm{cl}_{X} A$ is compact.

COROLlARY 5.5 (JUHÁSz). If $X$ is ultracompact regular, then $X$ is $\aleph_{0}$-bounded.

Question. What else can we say about when ultracompact implies $x_{0}$ bounded, or more generally, about when $\mathfrak{m}$-ultracompact implies $\mathfrak{m}$-bounded?

TheOREM 5.6. Assume that there exists $p \in \beta N \backslash N$ such that $p$ is not an accumulation point of any countable subset of $\beta N \backslash N$. Then there exists a Hausdorff space $X$ which is ultracompact but not $\aleph_{0}$-bounded.

Proof. Take $x \in \beta N \backslash N, x \neq p$. Let $X=\beta N \backslash\{p\}$. Set $\mathcal{T}=\{U \subset X$ : $U=\varnothing$ or $x \notin U$ and $U$ is open in $\beta N$ or $x \in U$ and $U=V \cup A$ with $x \in V$ open in $\beta N$ and $A \in p$ \}.

Then $X$ is a Hausdorff space, $X$ is not regular, $X$ is ultracompact since $\beta N$ is and the fiddling with $p$ does not affect ultracompactness since $p$ is not an accumulation point of any countable subset of $\beta N \backslash N$ and $x=p-$ $\lim _{n \rightarrow \infty} n$.

$X$ is not $\kappa_{0}$-bounded, that is, $X$ is not compact, because it is not regular and also because $X \backslash N$ is homeomorphic to $\beta N \backslash(N \cup\{p\})$, and is closed in $X$ but not compact.

REMARKS AND QUESTIONS. In [ $\left.\mathbf{B}_{2}\right] \mathrm{D}$. Booth shows that Martin's Axiom implies there exist $P$-points in $\beta N \backslash N$. Thus we have Martin's Axiom implies there exists a Hausdorff space which is ultracompact but not $\kappa_{0}$-bounded. Some natural questions arise. Is Martin's Axiom necessary to construct a point $p$ of $\beta N \backslash N$ which is not an accumulation point of any countable subset of $\beta N \backslash N$ ? Is Martin's Axiom necessary to construct a Hausdorff ultracompact space which is not $\kappa_{0}$-bounded?

ADDED IN PROOF. Kenneth Kunen has recently shown that there exist points of $\beta N \backslash N$ which are not accumulation points of any countable subset of $\beta N \backslash N$. Thus Theorem 5.6 shows that there exists a Hausdorff ultracompact space which is not $\aleph_{0}$-bounded. 
The construction in Theorem 5.6 can be generalized, that is, if one assumes that there exists $p \in \beta(\mathfrak{m}) \backslash \mathfrak{m}$ which is not an accumulation point of any subset of $\beta(\mathfrak{m}) \backslash \mathfrak{m}$ of cardinality $\leqslant \mathfrak{m}$, then there exists a Hausdorff $\mathfrak{m}$ ultracompact space which is not $\mathrm{m}$-bounded. This is not so interesting since the existence of such $p$ is still unknown; the question of their existence was asked in $\left[\mathbf{S S}_{1}\right]$. So we have another question: Does there exist $m>\aleph_{0}$ and a Hausdorff $\mathfrak{m}$-ultracompact space which is not $\mathfrak{m}$-bounded?

In [E, p. 165], it is stated that a theorem of Alexandroff and Urysohn in [AU] says that if $X$ is countably compact Hausdorff first countable, then $X$ is regular. The following theorem directly generalizes this result.

Definition. Let $X$ be a space. Then $\mathcal{X}(X)=$ the least cardinal such that each point of $X$ has a local basis of $\leqslant \mathscr{X}(X)$ open sets.

TheOREM 5.7. Let $X$ be Hausdorff $\left[\aleph_{0}, \mathfrak{m}\right]$-compact with $\mathcal{X}(X) \leqslant \mathfrak{m}$. Then $X$ is regular.

Proof. Take $x \in U$ open and we need to produce $O$ open with $x \in O \subset$ $\bar{O} \subset U$. Let $\left(O_{\xi}: \xi<\mathfrak{m}\right)$ be a local basis at $x$, and we assume without loss of generality that $O_{\xi} \subset U$, for all $\xi<\mathfrak{m}$. For each $y \in \bar{U} \backslash U$, there exists $O_{\xi}$ and $W_{\xi}^{y}$ open such that $O_{\xi} \cap W_{\xi}^{y}=\varnothing, x \in O_{\xi}$, and $y \in W_{\xi}^{y}$.

Let $B_{\xi}=\left\{y \in \bar{U} \backslash U:\right.$ there exists $W_{\xi}^{y}$ open, $x \in O_{\xi}, y \in W_{\xi}^{y}, W_{\xi}^{y} \cap O_{\xi}$ $=\varnothing\}$. For each $\xi<\mathfrak{m}$, let

$$
W_{\xi}=\bigcup\left\{W_{\xi}^{y}: y \in B_{\xi}\right\} .
$$

Then $\bar{U} \backslash U \subset \cup_{\xi<\mathrm{m}} W$ and so there exist $\xi_{1}, \ldots, \xi_{n}$ with $U \backslash U \subset$ $\cup_{i=1}^{n} W_{\xi}=W$.

Then $x \in O=\cap_{i=1}^{n} O_{\xi}$ is open and if $y \in \bar{U} \backslash U$, then $y \in W$ and $W \cap O=\varnothing$, so $y \notin \bar{O}$. Therefore $\bar{O} \subset U$.

An early version of this paper asked if there exists a countably compact Hausdorff space $X$ in which each point is a $G_{\delta}$ but $X$ is not first countable. In [A] C. E. Aull has constructed such a space.

6. $C[\mathfrak{n}, \mathfrak{m}]$ spaces. We first show where $C[\mathfrak{n}, \mathfrak{m}]$ fits into J. Vaughan's collection of properties in $\left[\mathbf{V}_{\mathbf{1}}\right]$.

Notation AND Definitions. If $S$ is a set, then

$$
\mathscr{P}_{\mathfrak{n}}(S)=\{A \subset S:|A|<\mathfrak{n}\} .
$$

A space $X$ has property $N[\mathfrak{n}, \mathfrak{m}]$ provided that every net in $X$ whose domain is $\mathscr{P}_{\mathfrak{n}}(\mathfrak{b})$ for some $\mathfrak{n} \leqslant \mathfrak{b} \leqslant \mathfrak{m}$ has a cluster point.

Vaughan shows that $N[\mathfrak{n}, \mathfrak{m}]$ implies $[\mathfrak{n}, \mathfrak{m}]$-compact and that they are equivalent if $\mathfrak{n}=\aleph_{0}$; in fact, all of his properties are equivalent if $\mathfrak{n}=\kappa_{0}$. We have already mentioned that a space is $\left[\kappa_{0}, \mathfrak{m}\right]$-compact if, and only if, it is $C\left[\aleph_{0}, m\right]$. The following proposition is useful in the verification of this result, and may also be of some interest in its own right. 
Proposition. Let $X$ be a noncompact space. Let a be the smallest infinite cardinal for which there exists a subset $A$ of $X$ of cardinality a which has no complete accumulation point. Then $a$ is a regular cardinal.

Proof. If $\mathfrak{a}$ is singular, then $\mathrm{cf}(\mathfrak{a})<\mathfrak{a}$, and we can write $A=\cup_{\xi<\mathrm{cf}(\mathfrak{a})} A_{\xi}$, where $\left|A_{\xi}\right|<\mathfrak{a}$ for each $\xi<\operatorname{cf}(\mathfrak{a})$, and the sets $\left(A_{\xi}: \xi<\operatorname{cf}(\mathfrak{a})\right)$ are pairwise disjoint. Then each $A_{\xi}$ has a complete accumulation point $p_{\xi}$, and the set $\left\{p_{\xi}\right.$ : $\xi<\operatorname{cf}(a)\}$ has a complete accumulation point $p$. Clearly $p$ is a complete accumulation point of $A$, which is the desired contradiction.

TheOREM 6.1. Let $X$ be a $C[\mathfrak{n}, \mathfrak{m}]$ space. Then $X$ is $[\mathfrak{n}, \mathfrak{m}]$-compact.

Proof. The proof is the same as the one outlined in Kelley [ $\left.\mathbf{K}_{3}, 5 \mathrm{I}\right]$.

We now show that $C[\mathfrak{n}, \mathfrak{m}]$ and $N[\mathfrak{n}, \mathfrak{m}]$ are independent properties, and that $[\mathfrak{n}, \mathfrak{m}]$-compact does not imply $C[\mathfrak{n}, \mathfrak{m}]$.

In $\left[\mathbf{V}_{\mathbf{1}}\right]$ it is shown that $N$ with the discrete topology is not $N\left[\boldsymbol{\kappa}_{\omega}, \mathfrak{b}\right]$, for any $\mathfrak{b} \geqslant \kappa_{\omega}$. But clearly $N$ is $C\left[\kappa_{\omega}, \mathfrak{b}\right]$ vacuously.

We now construct a space which is $N\left[\kappa_{1},-\right]$ but not $C\left[\aleph_{1},-\right]$, similar in flavor to spaces in $\left[\mathbf{M}_{\mathbf{1}}\right]$.

EXAMPLE. Let $X_{n}$ be a discrete space of cardinality $\kappa_{n}$, and let $X_{n}^{*}$ be the one point compactification of $X_{n}$.

Let $X=\bigoplus_{n \in N} X_{n}^{*}$, the disjoint union of the $X_{n}^{*}$. $X$ is $\sigma$-compact, hence Lindelöf, i.e. [ $\left.\boldsymbol{\kappa}_{1},-\right]$-compact. By $2 B$ of $\left[\mathbf{V}_{\mathbf{1}}\right] X$ is $N\left[\kappa_{1},-\right]$.

Now take any subset $A$ of $X$ with $|A|=\kappa_{\omega}$. Then since every point of $X$ has a neighborhood with contains fewer than $\aleph_{\omega}$ points, $A$ has no complete accumulation point in $X$, so $X$ is not $C\left[\aleph_{1},-\right]$.

We now characterize spaces all of whose powers are $C[\mathfrak{n}, \mathfrak{m}]$.

Definition. A space $X$ is $\left(\mathscr{D}_{\mathfrak{a}}: \mathfrak{n} \leqslant \mathfrak{a} \leqslant \mathfrak{m}\right)$-compact provided

(i) $\mathscr{D}_{\mathfrak{a}} \in \mu a$, for each $a$ with $\mathfrak{n} \leqslant \mathfrak{a} \leqslant \mathfrak{m}$;

(ii) if $\left(x_{\xi}: \xi<a\right)$ is a net in $X$, then $\left(x_{\xi}: \xi<a\right)$ has a $\mathscr{D}_{\mathfrak{a}}$-limit point in $X$.

Thus a space is $\left(\mathscr{D}_{\mathfrak{a}}: \mathfrak{n} \leqslant \mathfrak{a} \leqslant \mathfrak{m}\right)$-compact if, and only if, for each $\mathfrak{a}$ with $\mathfrak{n} \leqslant \mathfrak{a} \leqslant \mathfrak{m}$, the space is $\mathscr{D}_{\mathfrak{a}}-\mathfrak{a}$-compact.

THEOREM 6.2. Let $X$ be a space. Then all of the powers of $X$ are $C[\mathfrak{n}, \mathfrak{m}]$ if, and only if, there exists $\left(\mathscr{D}_{\mathfrak{a}}: \mathfrak{n} \leqslant \mathfrak{a} \leqslant \mathfrak{m}\right)$ for which $X$ is $\left(\mathscr{D}_{\mathfrak{a}}: \mathfrak{n} \leqslant \mathfrak{a} \leqslant \mathfrak{m}\right)$ compact.

Proof. Since a product of $\mathscr{D}_{\mathfrak{a}}-\mathfrak{a}$-compact spaces is $\mathscr{D}_{\mathfrak{a}}$ - $\mathfrak{a}$-compact, if $X$ is $\left(\mathscr{D}_{\mathfrak{a}}: \mathfrak{n} \leqslant \mathfrak{a} \leqslant \mathfrak{m}\right)$-compact, then all of its powers are $C[\mathfrak{n}, \mathfrak{m}]$.

Conversely, if there does not exist a family $\left(\mathscr{D}_{\mathfrak{a}}: \mathfrak{n} \leqslant \mathfrak{a} \leqslant \mathfrak{m}\right)$, then there exists $\mathfrak{a}$ with $\mathfrak{n} \leqslant \mathfrak{a} \leqslant \mathfrak{m}$ such that $X$ is not $\mathscr{D}-\mathfrak{a}$-compact, for any $\mathscr{D} \in \mu \mathfrak{a}$, so that there exists a net $\left(y_{\xi}^{\mathscr{D}}: \xi<a\right)$ with no $\mathscr{D}$-limit.

Take $\prod_{\mathscr{D} \in \mu \mathrm{a}} X_{\mathscr{D}} \times X_{i}=Y$ where each $X_{\mathscr{D}}=X_{i}=X$, and we take $\left(y_{\xi}\right.$ : $\xi<\mathfrak{a})$ defined by $\left(y_{\xi}\right)_{\mathscr{D}}=y_{\xi}^{\mathscr{D}}$ with $y_{i}$ such that $\left(y_{\xi}: \xi<a\right) \subset Y$ is faithfully 
indexed. Clearly $\left\{y_{\xi}: \xi<a\right\}$ has no complete accumulation point.

In [CS] and [SS $\mathbf{S S}_{1}$ it is shown that if $T$ is compact and $S \subset T$ with $|S| \leqslant \mathfrak{m}$, then there exists $X$ with $X \subset S \subset T, X$ is $C\left[\aleph_{0}, \mathfrak{m}\right]$, and $|X| \leqslant 2^{\mathfrak{m}}$. Note that if $T$ is Hausdorff and $|T|>2^{\mathfrak{m}}$, then $X$ is not compact. Moreover, if $T$ is a topological group, then $X$ may also be chosen to be a group. In [SV] it is shown that quite often $|X|=2^{\text {nt }}$.

We now wish to construct nontrivial $\left(\mathscr{D}_{\mathfrak{m}}: \mathfrak{n} \leqslant \mathfrak{a} \leqslant \mathfrak{m}\right)$-compact spaces. So for the remainder of this paper, $S$ is dense in $T$ compact Hausdorff, $|S| \leqslant \mathfrak{m}$, $|T|>2^{\mathfrak{m}}$, and for each $\mathfrak{a}$ with $\mathfrak{n} \leqslant \mathfrak{a} \leqslant \mathfrak{m}$, we have a fixed $\mathscr{D}_{\mathfrak{a}} \in \mu a$.

Notation. If $B \subset T$, set $E(B)=\left\{t \in T\right.$ : there exists $\left(b_{\xi}: \xi<a\right)$ a net from $B$ for which $\mathfrak{n} \leqslant \mathfrak{a} \leqslant \mathfrak{m}$ and $\left.t=\mathscr{D}_{\mathfrak{a}}-\lim _{\xi<\mathfrak{a}} b_{\xi}\right\}$. Also set $F(B)=B$ $\cup E(B)$.

As usual the proof is a transfinite induction over $\mathrm{m}^{+}$, and we first perform the inductive step as a lemma.

LeMma 6.3. Let $B \subset T$. If $|B| \leqslant 2^{\mathfrak{m}}$, then $|F(B)| \leqslant 2^{\mathfrak{m}}$.

Proof. Since $|B| \leqslant 2^{\mathfrak{m}}$, we have

$$
\left|\mathscr{P}_{m^{+}}(B)\right| \leqslant 2^{\mathfrak{m}} \text {. }
$$

Now if $C \in \mathscr{P}_{m^{+}}(B)$, then clearly $|E(C)| \leqslant 2^{\mathfrak{m}}$, since $C$ admits at most $2^{\mathrm{m}}$ well orderings according to a cardinal $a \leqslant \mathfrak{m}$ (admitting unfaithfully indexed nets) and since $T$ is Hausdorff, each such $a$-net has a unique $\mathscr{D}_{a}$-limit point.

Now $E(B)=\cup_{C \in \mathscr{P}_{m^{+}}(B)} E(C)$, and so $|E(B)| \leqslant 2^{\mathfrak{m}} 2^{\mathfrak{m}}=2^{\mathfrak{m}}$. It is now immediate that $|F(B)| \leqslant 2^{\mathfrak{m}}$.

THEOREM 6.4. There exists $X$ satisfying:

(i) $S \subset X \subset T$,

(ii) $|X| \leqslant 2^{\mathfrak{m}}$,

(iii) $X$ is $\left(\mathscr{D}_{\mathfrak{a}}: \mathfrak{n} \leqslant \mathfrak{a} \leqslant \mathfrak{m}\right)$-compact,

(iv) $X$ is not compact.

Moreover if $T$ is a topological group, then $X$ may also be chosen to be a group.

Proof. Let $X_{0}=S$, and for each $\xi<\mathfrak{m}^{+}$, define

$$
X_{\xi}=F\left(\bigcup_{\delta<\xi} X_{\delta}\right),
$$

and take $X=\cup_{\xi<\mathfrak{m}} X_{\xi}$. By Lemma 6.2 for each $\xi<\mathrm{m}^{+},\left|X_{\xi}\right| \leqslant 2^{\mathfrak{m}}$, and so $|X| \leqslant 2^{\mathfrak{m}}$. Clearly $S \subset X \subset T$ and since $X$ is a proper dense subset of $T, X$ is not compact.

To see that $X$ is $\left(\mathscr{D}_{\mathfrak{a}}: \mathfrak{n} \leqslant \mathfrak{a} \leqslant \mathfrak{m}\right)$-compact, let $\left(x_{\xi}: \xi<\mathfrak{a}\right)$ be a net from $X$ with $\mathfrak{n} \leqslant \mathfrak{a} \leqslant \mathfrak{m}$. Then there exists $\eta<\mathfrak{m}^{+}$with $\left\{x_{\xi}: \xi<\mathfrak{a}\right\} \subset X_{\eta}$. Thus there exists $x \in X_{\mathfrak{n}+1} \subset X$ such that $x=\mathscr{D}_{\mathfrak{a}}-\lim _{\xi<a} x_{\xi}$. 
If $T$ is a group, let $\langle B\rangle=$ the group generated by $B$ in $T$. Then take $X_{\xi}=\left\langle F\left(\cup_{\delta<\xi} X_{\delta}\right)\right\rangle$, and $X$ is a group which still enjoys properties (i) through (iv).

7. Questions. In this section we collect some of the questions scattered throughout this paper, and also include some other questions whose solution we would like to see.

1. Does there exist a collection of spaces $\left(X_{i}: i \in I\right)$ with $|I|=2^{c}$ such that $\Pi_{i \in I} X_{i}$ is not countably compact, but if $J \subset I$ with $|J|<2^{c}$, then $\Pi_{i \in J} X_{i}$ is countably compact?

2. Does there exist $\mathfrak{m}>\aleph_{0}$ for which there is a product of $\left[\aleph_{0}, \mathfrak{m}\right]$-compact spaces which is not $\left[\kappa_{0}, \mathfrak{m}\right]$-compact?

3. Is the product of countably compact topological groups countably compact?

4. Does there exist a separable Hausdorff ultracompact space which is not compact?

5. Is it consistent that there exists a space $X$ of cardinality $\leqslant c$ which is compact but not sequentially compact?

\section{REFERENCES}

[A] C. E. Aull, A certain class of topological spaces, Prace Mat. 11 (1967), 47-53.

[AU] P. Alexandroff and P. Urysohn, Mémoire sur les espaces topologiques compacts, Verh. Akad. Wetensch. Amsterdam 14 (1929), 1-96.

[B] A. R. Bernstein, A new kind of compactness for topological spaces, Fund. Math. 66 (1970), 185-193.

[B $\left.\mathbf{B}_{2}\right]$ D. Booth, Ultrafilters on a countable set, Ann. Math. Logic 2 (1970), 1-24.

[B $\mathbf{B}_{3}$ ] A. Blass, The Rudin-Keisler ordering. of P-points, Trans. Amer. Math. Soc. 179 (1973), 145-166.

[B] N. Bourbaki, Topologie générale, Hermann, Paris, 1965.

[BD] N. Bourbaki and J. Dieudonné, Note de la teratopologie. II, Rev. Scientifique 77 (1939), 180-181.

[C] W. W. Comfort, A nonpseudocompact product space whose finite subproducts are pseudocompact, Math. Ann. 170 (1967), 41-44.

[CN] W. W. Comfort and S. Negrepontis, The theory of ultrafilters, Springer-Verlag, Heidelberg, 1974.

[CS] W. W. Comfort and V. Saks, Countably compact groups and finest totally bounded topologies, Pacific J. Math. 49 (1973), 33-44.

[D] J. Dugundji, Topology, Allyn and Bacon, Boston, Mass., 1966.

[E] R. Engelking, Outline of general topology, North-Holland, Amsterdam, 1968.

[F $F_{1}$ Z. Frolik, Sums of ultrafilters, Bull. Amer. Math. Soc. 73 (1967), 87-91.

$\left[\mathrm{F}_{2}\right]$ _ On the topological product of paracompact spaces, Bull. Acad. Polon. Sci. Sér. Sci. Math. Astronom. Phys. 8 (1960), 747-750.

[GJ] L. Gillman and M. Jerison, Rings of continuous functions, Van Nostrand, Princeton, N. J., 1960.

[GS] J. Ginsburg and V. Saks, Some applications of ultrafilters in topology, Pacific J. Math. 57 (1975), 403-418.

[H] S. H. Hechler, On some weakly compact spaces and their products, General Topology and Appl. 5 (1975), 83-93. 
[HI] M. Henriksen and J. R. Isbell, Some properties of compactifications, Duke Math. J. 25 (1958), 83-106.

$\left[\mathbf{K}_{1}\right]$ K. Kunen, manuscript.

$\left[\mathbf{K}_{2}\right] \_$, Some points in $\beta N$, Math. Proc. Cambridge Philos. Soc. 80 (1976), 385-398.

[K $\mathbf{K}_{3}$ ] J. Kelley, General topology, Van Nostrand, New York, 1955.

[M] A. Mišcenko, Finally compact spaces, Soviet Math. J. 3 (1962), 1199-1202.

$\left[\mathbf{M}_{2}\right]$ P. Meyer, Sequential space methods in general topological spaces, Colloq. Math. 22 (1971), 223-228.

[RW] M. Rajagopalan and R. G. Woods, Products of sequentially compact spaces and the $V$-process, Trans. Amer. Math. Soc. (to appear).

[S $\left.\mathrm{S}_{1}\right]$ V. Saks, Countably compact groups, doctoral dissertation, Wesleyan University, 1972.

$\left[\mathbf{S}_{2}\right]$ _ Una demostración nueva del teorema de Tikonov, Bol. Mat. Costarricense (to appear).

[S $\mathbf{S}_{3}$ Y Yu. M. Smirnov, On topological spaces, compact in a given interval of powers, Izv. Akad. Nauk SSSR Ser. Mat. 14 (1950), 155-178.

$\left[\mathbf{S}_{4}\right]$ V. Saks, Ultrafilter invariants in topological spaces; abstract, Notices Amer. Math. Soc. 22 (1975), A-218.

[S $\left.\mathbf{S}_{5}\right]$ V. Saks, Perfect functions, Notices Amer. Math. Soc. 25 (1978), A-138.

[SS $]_{1}$ V. Saks and R. M. Stephenson, Jr., Products of m-compact spaces, Proc. Amer. Math. Soc. 28 (1971), 279-288.

[SS $]$ C. T. Scarborough and A. H. Stone, Products of nearly compact spaces, Trans. Amer Math. Soc. 124 (1966), 131-147.

[SS $\mathrm{SS}_{3}$ A. K. Steiner and E. F. Steiner, Relative types of points in $\beta N \backslash N$, Trans. Amer. Math. Soc. 160 (1971), 279-286.

[SV] R. M. Stephenson, Jr. and J. E. Vaughan, Products of initially m-compact spaces, Trans. Amer. Math. Soc. 196 (1974), 177-189.

$\left[V_{1}\right]$ J. Vaughan, Some properties related to $[a, b]$-compactness, Fund. Math. 87 (1975), 251-260.

$\left[\mathrm{V}_{2}\right] \longrightarrow$ Product spaces with compactness-like properties, Duke Math. J. 39 (1972), $611-617$.

$\left[\mathrm{V}_{3}\right] \ldots$, Products of perfectly normal sequentially compact spaces (to appear).

[W] R. C. Walker, The Stone-Cech compactification, Springer-Verlag, Heidelberg, 1974.

Universidad de Costa Rica, Ciudad Universitaria "Rodrigo Facio", Costa Rica

Current address: 681 Crown Street, Brooklyn, New York 11213 